\title{
Jordan tori and polynomial endomorphisms in $\mathbb{C}^{2}$
}

\author{
by
}

\author{
Manfred De nker and Stefan-M. H e i neman n (Göttingen)
}

\begin{abstract}
For a class of quadratic polynomial endomorphisms $f: \mathbb{C}^{2} \rightarrow \mathbb{C}^{2}$ close to the standard torus map $(x, y) \mapsto\left(x^{2}, y^{2}\right)$, we show that the Julia set $J(f)$ is homeomorphic to the torus. We identify $J(f)$ as the closure $\mathcal{R}$ of the set of repelling periodic points and as the Shilov boundary of the set $K(f)$ of points with bounded forward orbit. Moreover, it turns out that $(J(f), f)$ is a mixing repeller and supports a measure of maximal entropy for $f$ which is uniquely determined as the harmonic measure for $K(f)$.
\end{abstract}

1. Introduction. Ergodic theory for the dynamics of endomorphisms has been mostly studied for zero- and one-dimensional systems. This is often motivated by number-theoretical problems, reduction of flows to (Poincaré) sections or coding problems. Only recently, attempts have been made to develop a theory for one-dimensional complex systems (see e.g. [1]). Whereas the generalisation of number-theoretical transformations to higher dimensions has been partly successful (see [21]), only a few results on the ergodictheoretic aspects of higher-dimensional endomorphisms have been published (e.g. [17]).

In this paper we study quadratic polynomial mappings $f$ of the form

$$
\left(\begin{array}{l}
x \\
y
\end{array}\right) \mapsto\left(\begin{array}{c}
p(x, y) \\
q(x, y)
\end{array}\right):=\left(\begin{array}{c}
x^{2}+k(y) \\
y^{2}+l(x)
\end{array}\right)
$$

where $k$ and $l$ are quadratic polynomials with complex coefficients satisfying, for some $\varepsilon>0$,

$$
\sup _{|x| \leq 1 / 2+\sqrt{1 / 2-\varepsilon}} \max \{|k(x)|,|l(x)|\} \leq 1 / 4-\varepsilon .
$$

These maps are called torus maps (see Definition 3.3). One of the basic problems in order to clarify the ergodic-theoretic behaviour of a dynamical system is to prove or disprove the existence of attractors (repellers, resp.).

1991 Mathematics Subject Classification: 32F05, 32H50, 58F11, 58F12. 
Here we show the existence of an expanding repeller homeomorphic to the torus $|x|=|y|=1$, with $f$ topologically conjugate to the standard map on the torus. We identify the repeller as the closure $\mathcal{R}$ of the set of repelling periodic points and provide other characterisations.

From [15] we recall the notion of weak normality:

Definition. A family $\left\{f_{k}: U \rightarrow \mathbb{C}^{n}\right\}$ of holomorphic functions on a domain $U \subseteq \mathbb{C}^{n}$ is called weakly normal at a point $z \in U$ if there is an open neighbourhood $V$ of $z$ and a family $\left\{\mathcal{C}_{x}\right\}$ of at least one-dimensional (complex) analytic sets indexed by the points $x \in V$, such that each $x$ lies in the corresponding analytic set $\mathcal{C}_{x}$ and, for each $x \in V$, the family $\left\{f_{k}\right\}$ restricted to $\mathcal{C}_{x} \cap V$ is normal (including convergence to infinity).

Throughout the paper $\|\cdot\|$ denotes the maximum norm on $\mathbb{C}^{2}$, hence the ball $B_{r}$ of radius $r$ equals the poly-disk $P_{r}$ of radius $r$.

Our main result is the following.

TheOREM. For a torus map $f: \mathbb{C}^{2} \rightarrow \mathbb{C}^{2}$ the following sets are equal:

(A) the Julia set $J(f):=\left\{z \in \mathbb{C}^{2}:\left\{f^{k}: k \geq 0\right\}\right.$ is not weakly normal at $z$;

(B) the Shilov boundary $\partial_{\mathrm{SH}} K(f)$, where

$$
K(f):=\left\{z \in \mathbb{C}^{2}: \sup _{k}\left\|f^{k}(z)\right\|<\infty\right\} ;
$$

(C) the closure $\mathcal{R}$ of the set of repelling periodic points;

(D) the limit of the pull backs $f^{-k}\left(\partial_{\mathrm{SH}} \bar{B}_{r}\right)$ for sufficiently large $r>0$;

(E) the support of the harmonic measure $\mu_{K}$ for $K(f)$ which has maximal entropy (for $f$ ).

The strategy to prove this result is by using iteration theory of holomorphic maps in dimension $\geq 2$. This has been developed for the case of polynomial automorphisms of $\mathbb{C}^{2}$, so-called complex Hénon maps (see e.g. [3]). There are also investigations of endomorphisms of complex projective spaces, hence homogeneous polynomial mappings, for example [5]. One might investigate the dynamics of skew products in $\mathbb{C}^{2}$ (cf. [15]). Here we use a different approach and consider polynomial endomorphisms of complex spaces in general (at least as general as possible) and look for characteristics of their dynamical behaviour. Hence, besides the above mentioned theorem we study basic, non-trivial dynamical properties of these maps.

In Section 2 we describe the set of polynomial mappings $f: \mathbb{C}^{n} \rightarrow \mathbb{C}^{n}$ for arbitrary $n \in \mathbb{N}$ which satisfy a growth condition similar to that for polynomials in $\mathbb{C}^{1}$. These maps are called strict polynomials (see [13]).

It turns out that, for a strict polynomial $f: \mathbb{C}^{n} \rightarrow \mathbb{C}^{n}$, the set $K(f)$ is compact, hence it makes sense to investigate the different definitions for the Julia set mentioned in the theorem. We first discuss this for the standard 
torus map $\sigma_{2}:(x, y) \mapsto\left(x^{2}, y^{2}\right)$. In particular, we define the Julia set using weakly normal convergence. For $n=1$, normal convergence and weakly normal convergence are equivalent. Furthermore, for the special case of $n$ vectors of polynomials of one variable (including the standard torus map), we show that the Julia set in $\mathbb{C}^{n}$ is exactly the product of the associated onedimensional Julia sets. As a direct consequence we obtain the equivalence of the different definitions in this case.

In the last and main part of this paper (Section 3) we prove the theorem stated above. In the course of proof we also derive some theorems and corollaries of independent interest. We show that the Julia sets of torus maps are homeomorphic to the torus. This is established by splitting $\mathbb{C}^{2}$ into subsets $G_{0}, G_{\infty}, G_{1 x}, G_{1 y}$, and $G_{1 x y}$ (see Figure 1 in Section 3). We show (weak) normality of $\left(f^{n}\right)$ on $G_{0}$ and $G_{\infty}$, then we find an invariant compact set $(\partial K)^{*} \subset G_{1 x y}$ which turns out to be homeomorphic to the torus. We then prove that $(\partial K)^{*}$ is equal to $\partial_{\mathrm{SH}} K(f)$ (it makes sense to define the Shilov boundary for $K$ instead of for the space $\mathcal{A}$ of maximal ideals of the algebra $\operatorname{Hol}(K(f))$, because $K(f)$ is polynomially convex and hence $K(f)$ is isomorphic to $\mathcal{A}$ (cf. [8])). Weak normality is established in $G_{1 x} \cup G_{1 y} \cup\left(G_{1 x y} \backslash(\partial K)^{*}\right)$, as, for each $z \in \partial K \backslash(\partial K)^{*}$, we find a stability set given by a complex analytic curve $\mathcal{C}_{z}$. Thus $(\partial K)^{*}$ also equals the Julia set $J(f)$. The dynamics of $f$ on $J(f)$ gives a mixing repeller conjugate to $\sigma_{2}$, in particular, $J(f)$ is the support of a measure of maximal entropy for $f$.

Acknowledgements. We would like to thank the referee for his valuable comments which improved the paper considerably.

Dedication. Diese Arbeit ist in besonderem Maße Wiesław Szlenk gewidmet. Wir schätzen uns glücklich, daß wir einige wesentliche Ideen für diese Arbeit mit Wiesław bei seinem letzten Besuch in Göttingen diskutieren konnten. Der erstgenannte Autor war mit Wiesław Szlenk mehr als 25 Jahre verbunden. In dieser Zeit sind viele neue Entwicklungen in der Dynamik in Polen und Deutschland von Wiesław aktiv gefördert worden. So hat er auch seinen Anteil an dem hier vorgestellten Konzept einer Dynamik holomorpher Abbildungen in $\mathbb{C}^{n}$.

2. Product maps. In this section we briefly recall some basic facts from [13], [14] and [15]. We also derive some general results on the dynamics of strict polynomials and illustrate the method of proof of the main theorem for product maps. In particular, Theorem 2.6 below gives the main theorem for the case of product maps in any dimension. The proofs are only given for the results which are neither direct modifications of their counterparts in dimension one nor immediate consequences of the growth condition (1) given below. 
Recall ([13], Ch. 1) that an entire mapping $f: \mathbb{C}^{n} \rightarrow \mathbb{C}^{n}$ is called a strict polynomial of degree $p \in \mathbb{N}$ if, for some $k_{1}, k_{2}>0, r \in \mathbb{R}$,

$$
k_{1}\|z\|^{p} \leq\|f(z)\| \leq k_{2}\|z\|^{p} \quad \text { for }\|z\|>r .
$$

For future use we define

$$
R_{f}:=\max \left\{r, 1 / \sqrt[p-1]{k_{1}}\right\}
$$

and note that $\|z\|>R_{f}$ implies $\|f(z)\|>\|z\|$. As in dimension 1 we deduce from the growth condition that the composition of strict polynomials is a strict polynomial. In particular, all iterates $f^{k}$ (for $k \in \mathbb{N}$ ) of a strict polynomial $f: \mathbb{C}^{n} \rightarrow \mathbb{C}^{n}$ are also strict (of degree $p^{k}$ ). It is clear that strict polynomials are proper mappings, hence are compatible with the Alexandrov compactification and admit a continuation to $\overline{\mathbb{C}^{n}}:=\mathbb{C}^{n} \cup\{\infty\}$ by setting $f(\infty):=\infty$. We note that the attracting basin $F_{\infty}:=F_{\infty}(f)$ for "infinity", i.e. the set of points whose forward orbits eventually leave any compact set in $\mathbb{C}^{n}$ ("converge to infinity"), is not empty.

Applying Bézout's theorem it has been shown in [13] that a strict polynomial $f: \mathbb{C}^{n} \rightarrow \mathbb{C}^{n}$ is surjective and has mapping degree $p^{n}$. It immediately follows that a strict polynomial has $p^{k n}$ periodic points of order $k \in \mathbb{N}$ (counted with multiplicity), hence the set $K(f)$ is not empty. Only $[f]$, the terms of maximal degree of $f$, are relevant to the strictness of $f$. This shows that the strict polynomials are dense in the parameter space of polynomials of a given (algebraic) degree. In this context we note that condition (1) can be weakened to yield an even larger class of endomorphisms, the so-called $(p, q)$-regular mappings (cf. [14], Ch. 2). Namely, we require that, for some $p \in \mathbb{Q}, q \in \mathbb{N}, k_{1}, k_{2}>0, r \in \mathbb{R}$,

$$
k_{1}\|z\|^{p} \leq\|f(z)\| \leq k_{2}\|z\|^{q} \text { for }\|z\|>r .
$$

The reader may check that, with the exception of the immediate application of Bézout's theorem and the direct calculation of the Green function, the results of this paper can be generalised to families of $(p, q)$-regular mappings (e.g. maps close to $\sigma_{a, b}:(x, y) \mapsto\left(x^{a}, y^{b}\right)$, where $\left.a, b \geq 2\right)$.

REMARK 2.1. It is clear that we should expect the Julia set $J(f)$ to be contained in $\partial K(f)$. This is simply an application of Cauchy's integral formula which has a complete analogue in higher dimensions ([9], p. 13).

We prepare our investigation of the Julia sets of general torus maps by a brief discussion of maps of product type. Recall that the Julia set $J(f)$ of a strict polynomial is defined to be the set of points where $\left\{f^{k}\right\}$ is not weakly normal (see [13]). We refer to its complement as the Fatou set $F(f)$ of $f$. 
Definition 2.2 (standard torus map). Let us define the standard torus map $\sigma_{2}: \mathbb{C}^{2} \rightarrow \mathbb{C}^{2}$ by

$$
\sigma_{2}:\left(\begin{array}{l}
x \\
y
\end{array}\right) \mapsto\left(\begin{array}{c}
x^{2} \\
y^{2}
\end{array}\right)
$$

It is obvious that

$$
K\left(\sigma_{2}\right)=\overline{\mathbb{B}} \times \overline{\mathbb{B}} .
$$

Further, in view of Remark 2.1, we see that

$$
\begin{aligned}
\partial K\left(\sigma_{2}\right) & =\left(S^{1} \times S^{1}\right) \dot{\cup}\left(\mathbb{B} \times S^{1}\right) \dot{\cup}\left(S^{1} \times \mathbb{B}\right) \\
& =\left\{\text { points where }\left(f^{k}\right) \text { is not normal convergent } .\right.
\end{aligned}
$$

Each of the sets in (2) is completely invariant. The last two are not compact, but contain the compact invariant sets $\{0\} \times S^{1}, S^{1} \times\{0\}$, resp. We see that $f$ restricted to either of

$$
S^{1} \times S^{1}, \quad\{0\} \times S^{1}, \quad S^{1} \times\{0\}
$$

gives rise to a topologically mixing system, but only the subsystem $\left.f\right|_{S^{1} \times S^{1}}$ has maximal topological entropy $2 \log (2)$ (cf. [11]), whereas the others yield $\log (2)$ (each obtained by considering suitably normalised Lebesgue measure). From the point of view of the ergodic-theoretic behaviour of one-dimensional complex polynomials the set $S^{1} \times S^{1}$ should be contained in the Julia set of $\sigma_{2}$. Since one-dimensional Julia sets are equal to the Shilov boundary of $K(f)$, we would like to investigate the relation of $S^{1} \times S^{1}$ and $\partial_{\mathrm{SH}} K\left(\sigma_{2}\right)$. To do so we need an additional definition.

Definition 2.3. A set $D \subset \mathbb{C}^{n}$ is called a Weil analytic polyhedron if it can be defined in terms of finitely many holomorphic functions $\varphi_{i}: G \rightarrow \mathbb{C}$, $i=1, \ldots, N$, on a domain $G \subseteq \mathbb{C}^{n}$ with $\bar{D} \subset G$, by

$$
D:=\left\{z \in G:\left|\varphi_{i}(z)\right|<1 \text { for all } i\right\} .
$$

We call the set

$$
\Sigma(D):=\left\{z \in G:\left|\varphi_{i}(z)\right|=1 \text { for all } i\right\}
$$

the skeleton of $D$.

With this terminology we obtain the following result.

Theorem 2.4 ([7], Th. 15.4). Let $D$ be a Weil analytic polyhedron defined above. If for each vector $\chi:=\left(\chi_{1}, \ldots, \chi_{n}\right) \in\left(S^{1}\right)^{n}$ and each set of indices $1 \leq i_{1}<\ldots<i_{n} \leq N$ the set $\Sigma(\chi):=\left\{z \in G: \varphi_{i_{j}}(z)=\chi_{i_{j}}\right.$ for all $\left.i_{j}\right\}$ consists of isolated points, then

$$
\partial_{\mathrm{SH}} \bar{D}=\Sigma(D) \text {. }
$$

Evidently, $\stackrel{\circ}{K}^{\circ}\left(\sigma_{2}\right)=\mathbb{B} \times \mathbb{B}$ is a Weil analytic polyhedron defined by the coordinate functions $\varphi_{1}(x, y):=x, \varphi_{2}(x, y):=y$. It satisfies the conditions 
of Theorem 2.4 as $\Sigma\left(\chi_{1}, \chi_{2}\right)=\left\{\left(\chi_{1}, \chi_{2}\right)\right\}$. We deduce that

$$
\partial_{\mathrm{SH}} K\left(\sigma_{2}\right)=\Sigma(\mathbb{B} \times \mathbb{B})=S^{1} \times S^{1} .
$$

The reader should note that this standard fact can be established directly by using the fact that $K\left(\sigma_{2}\right)$ is a product set. However, the above proof carries over to the general setting in 3.9.

For the standard torus map $\sigma_{2}$, we note that the set of points where the sequence of iterates of $\sigma_{2}$ is not normal convergent coincides with (2). However, for $\left(x^{*}, y^{*}\right)$ in $S^{1} \times \mathbb{B}\left(\right.$ resp. in $\left.\mathbb{B} \times S^{1}\right)$, there still is some convergent behaviour. Namely $\left\{f^{k}\right\}$ restricted to $\left\{x^{*}\right\} \times \mathbb{B}$ (resp. restricted to $\mathbb{B} \times\left\{y^{*}\right\}$ ) is a normal family. Moreover, this also holds for any $\left(x^{\prime}, y^{\prime}\right)$ in the open neighbourhood $\mathbb{C} \times \mathbb{B} \cup \mathbb{B} \times \mathbb{C}$ of these $\left(x^{*}, y^{*}\right)$.

This shows that $\left\{f^{k}\right\}$ is weakly normal on $\mathbb{C}^{2} \backslash S^{1} \times S^{1}$. Evidently, $J\left(\sigma_{2}\right)$ must be a closed set according to the definition of weak normality. One cannot obtain normal convergence on any (at least one-dimensional) analytic set containing one of the repelling periodic points $\left(\exp \left(2 \pi i \cdot r /\left(2^{k}-1\right)\right)\right.$, $\left.\exp \left(2 \pi i \cdot s /\left(2^{k}-1\right)\right)\right), r, s \in \mathbb{N}, k \in \mathbb{N}^{*}$, of $f$. But these points are dense in $S^{1} \times S^{1}$. We conclude that $J\left(\sigma_{2}\right)=S^{1} \times S^{1}$.

For the reader's convenience, we recall some basic properties of the Julia set from [13]. $J(f)$ is closed and contained in $\partial K(f)$, hence compact. Applying the Open Mapping Theorem ([10], p. 108) and the Proper Mapping Theorem ([10], p. 213) one can show that $J(f)$ is completely invariant under $f$. For $n=1$, we remark that weakly normal and normal convergence give the same result as one-dimensional analytic sets are just open sets in $\mathbb{C}$.

In the final part of this section we investigate the behaviour of products of one-dimensional maps. For $n$ polynomials $f_{i}: \mathbb{C} \rightarrow \mathbb{C}$ in one variable, where $\operatorname{deg}\left(f_{i}\right)=p_{i} \geq 2$, we define a polynomial vector $f: \mathbb{C}^{n} \rightarrow \mathbb{C}^{n}$ by setting

$$
f\left(z_{1}, \ldots, z_{n}\right):=\left(f_{1}\left(z_{1}\right), \ldots, f_{n}\left(z_{n}\right)\right) .
$$

Evidently, $f$ is $(p, q)$-regular, where $p=\min _{i} p_{i}$ and $q=\max _{i} p_{i}$. Let $J\left(f_{i}\right)$ $\subset \mathbb{C}$ denote the Julia set of each $f_{i}: \mathbb{C} \rightarrow \mathbb{C}$. The following theorem holds.

Theorem 2.5. For $f: \mathbb{C}^{n} \rightarrow \mathbb{C}^{n}$ as above,

$$
J(f)={\underset{X=1}{n}}_{i=1}^{n} J\left(f_{i}\right) .
$$

P r o of. In view of the preceding example, it suffices to show that we get normal convergence on the sets

$$
\partial K_{(i)}:=\left\{\left(z_{1}, \ldots, z_{n}\right) \in \partial K(f): z_{i} \in \stackrel{\circ}{K}\left(f_{i}\right)\right\}
$$

(which might be empty for $\partial K\left(f_{i}\right)=K\left(f_{i}\right)$, e.g. for $J\left(f_{i}\right)$ a Cantor set), 
which implies

$$
J(f) \subseteq \partial K(f) \backslash \bigcup_{i=1}^{n} \partial K_{(i)}={\underset{i=1}{n}}^{n} J\left(f_{i}\right) .
$$

Furthermore, the repelling periodic points of $f$ are dense in $\chi_{i=1}^{n} J\left(f_{i}\right)$. By the same argument as in the case of $\sigma_{2}$ we obtain

$$
J(f) \supseteq \chi_{i=1}^{n} J\left(f_{i}\right) .
$$

We now show the main theorem in the case of product maps. We can characterise the set $J(f)$ in various ways.

TheOREM 2.6. In the product case we obtain (let $\left.r \geq R_{f}\right)$ :

$$
\begin{aligned}
J(f) & =\left\{z \in \mathbb{C}^{n}:\left(f^{k}\right) \text { is not weakly normal at } z\right\} \\
& =\partial_{\mathrm{SH}} K(f) \\
& =\{z: z \text { is a repelling periodic point of } f\} \\
& =\lim _{k \rightarrow \infty} f^{-k}\left(\partial_{\mathrm{SH}} \bar{B}_{r}\right) \\
& =\operatorname{supp}\left(\text { measure } \mu^{f} \text { of maximal entropy for } f\right) .
\end{aligned}
$$

Proof. First note that by Theorem 2.5 the form (A) is equivalent to

$$
J(f)=\underset{i=1}{\chi_{1}^{n}} J\left(f_{i}\right) .
$$

We show that $\left(\mathrm{A}^{\prime}\right)$ is equivalent to $(\mathrm{B})$ by using the following well known fact from dimension one: A point $z^{*} \in K(f)$ lies in $\partial_{\mathrm{SH}} K(f)$ if and only if for each neighbourhood $U \ni z^{*}$ there exists a peak function $\varphi_{U} \in \mathbb{A}$ such that $\left|\varphi_{U}\right|$ has its maximum in $U$ but takes only smaller values on $C U$ (for the notation, see [15]).

In view of this we have to show that for points $z^{*} \in J(f)$ and open sets $U \ni z^{*}$ there exist peak functions $\varphi_{U}: \mathbb{C}^{n} \rightarrow \mathbb{C}$, and that for $z^{*} \notin J(f)$ one cannot find $\varphi_{U}$ for arbitrary $U \ni z^{*}$. For one-dimensional maps $f_{i}$ we know $J\left(f_{i}\right)=\partial_{\mathrm{SH}} K\left(f_{i}\right)$, hence for $z_{i}^{*} \in U_{i} \subset \mathbb{C}$ we can find appropriate peak functions $\varphi_{U_{i}}: K\left(f_{i}\right) \rightarrow \mathbb{C}$. We may assume that $U$ is given in the form $\prod_{i} U_{i}$ and set $\varphi_{U}(z):=\prod_{i} \varphi_{U_{i}}\left(z_{i}\right)$. This shows $J(f) \subseteq \partial_{\mathrm{SH}} K(f)$. The remaining points $z^{*} \in \partial K(f)$ lie in sets $\partial K_{(i)}$. If we consider the mapping $\varphi_{U}$ restricted to

$$
\left\{z_{1}^{*}\right\} \times \cdots \times K\left(f_{i}\right) \times \cdots \times\left\{z_{n}^{*}\right\}
$$

we see by the maximum principle that it takes its maximum in

$$
\left\{z_{1}^{*}\right\} \times \cdots \times \partial K\left(f_{i}\right) \times \cdots \times\left\{z_{n}^{*}\right\}
$$


hence by iterating the argument we see that any $\varphi_{U}$ takes its maximal modulus in

$$
\partial K\left(f_{1}\right) \times \cdots \times \partial K\left(f_{n}\right)=J(f),
$$

thus $\partial_{\mathrm{SH}} K(f) \supseteq J(f)$.

For the equivalence of $\left(\mathrm{A}^{\prime}\right)$ and $(\mathrm{C})$ observe that $z^{*}$ is a repelling periodic point of $f$ if and only if each component $z_{i}^{*}$ is a repelling periodic point for $f_{i}$.

In order to prove the equivalence of $\left(\mathrm{A}^{\prime}\right)$ and $(\mathrm{D})$ we note (letting $\pi_{i}$ : $\mathbb{C}^{n} \rightarrow \mathbb{C}$ be the projection to the $i$ th coordinate)

$$
\pi_{i}\left(f^{-k}\left(\partial_{\mathrm{SH}} \bar{B}_{r}\right)\right)=f_{i}^{-k}\left(\pi_{i}\left(\partial_{\mathrm{SH}} \bar{B}_{r}\right)\right) .
$$

Using [4], Theorem 16.1, we handle (E) in a similar fashion:

$$
\mu_{k}^{f}(z):=\mu_{k}^{f_{1}}\left(z_{1}\right) \otimes \cdots \otimes \mu_{k}^{f_{n}}\left(z_{n}\right) .
$$

3. Torus maps. In dimension one it is well known that the Julia sets of mappings of the form

$$
f_{c}: z \mapsto z^{2}+c
$$

with $|c|$ small are Jordan curves (see [2], 1.6, 9.9, and [4], Th. 8.1) and exhibit dynamical behaviour similar to $\sigma_{1}: z \mapsto z^{2}$. It is easy to see that this holds if $|c|<1 / 4$. We want to obtain a similar result concerning strict polynomials $f: \mathbb{C}^{2} \rightarrow \mathbb{C}^{2}$ with respect to $\sigma_{2}$. We are interested in sharp results, i.e. we do not just want to establish the existence of some tiny epsilon-ball in parameter space where $f$ exhibits similar behaviour, but would like to obtain a "large" set in the sense that one might actually "see" the results using numerical approximation.

We proceed in several steps. First we show that the dynamics of $f$ induces a repeller $(\partial K)^{*} \subset \partial K(f)$ which is homeomorphic to the torus $S^{1} \times S^{1}$. The Shilov boundary of $K(f)$ is contained in $J(f)$ (this is part of (B)). But $\partial_{\mathrm{SH}} K(f)$ is also contained in $(\partial K)^{*}$. Hence, in the next step we show the equality of $(\partial K)^{*}$ and $\partial_{\mathrm{SH}} K(f)$. We then prove that the sequence $\left(f^{n}\right)$ is weakly normal on $\partial K(f) \backslash(\partial K)^{*}$. As $\partial_{\mathrm{SH}} K(f)$ is the support of the measure $\mu_{K}$ induced by the Green current (which has maximal entropy) we have the equivalence of (A), (B), (D), (E). Finally, we show that there are no repelling periodic points outside $G_{1 x y}$ but that they are dense in $J(f)$, which gives equivalence to $(\mathrm{C})$.

First let us assure the reader that we are not dealing with empty "phantom sets" $J(f)$.

THEOREM 3.1. For a strict polynomial $f: \mathbb{C}^{n} \rightarrow \mathbb{C}^{n}$ the Julia set $J(f)$ contains the Shilov boundary $\partial_{\mathrm{SH}} K(f)$ of $K(f)$.

Proof. Let $z^{*} \in \partial K(f) \backslash J(f)$. Hence, there exists an open set $V$ and complex analytic sets $\left\{\mathcal{C}_{x}\right\}$ such that $\left\{f^{k}\right\}$ restricted to $V \cap \mathcal{C}_{x}$ is normal. 
Now we fix an open set $U \ni z^{*}$ with $U \Subset V$ and assume the existence of a peak function $\varphi_{U}$ for $U$. By the maximum principle for complex spaces with boundary (see [10], p. 110) $\varphi_{U}$ restricted to $\bar{U} \cap \mathcal{C}_{x}$ for $x \in \bar{U}$ takes its maximal modulus in $\partial U \cap \mathcal{C}_{x}$, hence $z^{*} \notin \partial_{\mathrm{SH}} K(f)$ and thus

$$
\partial_{\mathrm{SH}} K(f) \subseteq J(f) .
$$

Corollary 3.2. For a strict polynomial $f: \mathbb{C}^{n} \rightarrow \mathbb{C}^{n}$ the Julia set $J(f)$ is not empty.

Consider an arbitrary strict polynomial $f: \mathbb{C}^{2} \rightarrow \mathbb{C}^{2}$ of (algebraic) degree 2 . It is given by a complex polynomial vector of the form

$$
f:\left(\begin{array}{l}
x \\
y
\end{array}\right) \mapsto\left(\begin{array}{c}
a x^{2}+b x y+c y^{2}+d x+e y+f \\
A y^{2}+B x y+C x^{2}+D y+E x+F
\end{array}\right) .
$$

Strictness of $f$ only depends on $[f]$, namely $f: \mathbb{C}^{2} \rightarrow \mathbb{C}^{2}$ is strict if and only if $[f]$ induces an endomorphism of $\mathbb{P}^{1}$ of the same algebraic degree. In the special case (4) this means that the one-dimensional rational map

$$
\tilde{f}: z \mapsto \frac{a z^{2}+b z+c}{A+B z+C z^{2}}
$$

has mapping degree 2 (cf. [22], 1.§4). This is easily checked by calculating the Sylvester determinant (cf. [20], p. 38)

$$
S(f):=(a A-c C)^{2}-(a B-b C)(A b-B c) .
$$

If $S(f) \neq 0$ then $f$ is strict of degree 2 .

We can apply linear mappings from $\operatorname{GL}(2, \mathbb{C})$ and translations in $\mathbb{C}^{2}$ as conjugation mappings to obtain normal forms of strict polynomials $f: \mathbb{C}^{2} \rightarrow$ $\mathbb{C}^{2}$. Evidently, in our case, the coefficients of $[f]$ are only affected by conjugation with members of $\mathrm{GL}(2, \mathbb{C})$. If we interpret this in the projective space $\mathbb{P}^{1}$ these maps correspond exactly to the holomorphic Möbius transforms on the Riemann sphere. Let us recall that they operate threefold transitive on $\mathbb{P}^{1}([16]$, p. 65$)$. We will make use of this in order to obtain a suitable normal form.

If $f$ is strict of degree 2 the Riemann-Hurwitz theorem ([6], p. 128) gives us the existence of exactly 2 different critical values $\lambda_{1}$ and $\lambda_{2}$ (and also 2 critical points $c_{1}, c_{2}$ ) of $\widetilde{f}$. Because of the threefold transitivity of the Möbius group on $\mathbb{P}^{1}$ we can move the critical points of $\tilde{f}$ to 0 and $\infty$ (then the critical values become $c / A, a / C$, resp.) by conjugation with a suitable Möbius map. Translation back to $\mathbb{C}^{2}$ and $[f]$ tells us that we can always find a $\operatorname{GL}(2, \mathbb{C})$-mapping such that conjugation of $[f]$ with this map gives another homogeneous map $[f]^{*}$ such that the planes $\mathbb{C} \cdot(0,1)(\cong$ " 0 " $), \mathbb{C} \cdot(1,0)$ 
$(\cong$ " $\infty$ ") are the only inverse images of their image planes $\mathbb{C} \cdot(c, A), \mathbb{C} \cdot(a, C)$ resp.

$\lambda_{1}$ and $\lambda_{2}$ are exactly those parameter values $(\in \overline{\mathbb{C}})$ for which $\widetilde{f}(z)=\lambda$ has a double root. This is equivalent to (if one $\lambda_{i}$ is $\infty$, we conjugate with $z \mapsto 1 / z)$

$$
(a-\lambda C)(c-\lambda A)=(b-\lambda B)^{2} .
$$

For our normal form we want to achieve that the critical points are 0 and $\infty$, hence the critical values are $c / A$ and $a / C$. Together with (6) this implies

$$
A b-c B=0, \quad a B-C b=0 .
$$

Strictness of $f$ and the form of $S(f)$ (see (5)) show that in this case

$$
0 \neq S(f)=(a A-c C)^{2}
$$

hence $(a, C)$ and $(c, A)$ are linearly independent and (7) can only be valid if $b=B=0$. This shows that a strict polynomial of degree 2 in $\mathbb{C}^{2}$ is conjugate to a map of the form

$$
f:\left(\begin{array}{l}
x \\
y
\end{array}\right) \mapsto\left(\begin{array}{c}
a x^{2}+c y^{2}+d x+e y+f \\
A y^{2}+C x^{2}+D y+E x+F
\end{array}\right) .
$$

We want to consider maps similar to the standard torus map $\sigma_{2}$, hence let us assume that $a A \neq 0$ in (8). Further conjugation by translation yields our final normal form

$$
f:\left(\begin{array}{l}
x \\
y
\end{array}\right) \mapsto\left(\begin{array}{c}
x^{2}+c y^{2}+e y+f \\
y^{2}+C x^{2}+E x+F
\end{array}\right) .
$$

We use the abbreviations

$$
k(y):=c y^{2}+e y+f, \quad l(x):=C x^{2}+E x+F .
$$

$k(y)$ and $l(y)$ correspond to the constant $c$ in (3). Of course it would be useless to demand that they are bounded for all $x$ and $y$. This would imply that $k$ and $l$ are constants and $f$ a product mapping. We could simply apply Theorem 2.6. Since we know that $\complement \bar{B}_{R_{f}} \subset F_{\infty}$, it is sufficient to control $k$ and $l$ on $\bar{B}_{R_{f}}$.

We recall the definition of a torus map (cf. the introduction).

Definition 3.3 (torus map). A quadratic strict polynomial $f: \mathbb{C}^{2} \rightarrow \mathbb{C}^{2}$ given by (9) is called a torus map if, for some $\varepsilon>0$, we have

$$
\left.\begin{array}{l}
\|k(y)\|_{B_{1 / 2+\sqrt{1 / 2-\varepsilon}}} \\
\|l(x)\|_{B_{1 / 2+\sqrt{1 / 2-\varepsilon}}}
\end{array}\right\} \leq 1 / 4-\varepsilon .
$$

REMARK 3.4. Throughout this paper we require that the quantity $\varepsilon$ is, in addition, bounded from below by $\varepsilon_{2}>37 / 150$ (this is due to the evaluation 
of (32) and (33) where we use the original map rather than iterates of higher degree in Theorem 3.15). This bound can easily be improved by using more detailed estimates.

We shall later see that in this case $1 / 2+\sqrt{1 / 2-\varepsilon}$ plays the role of $R_{f}$. We define some useful abbreviations:

$$
\begin{gathered}
\kappa:=1 / 4-\varepsilon, \quad \varrho:=1 / 2+\sqrt{\varepsilon}, \quad \varrho^{\prime}:=1 / 2-\sqrt{\varepsilon}, \\
r:=1 / 2+\sqrt{1 / 2-\varepsilon} .
\end{gathered}
$$

Note that

$$
\begin{gathered}
\varrho^{\prime 2}=\varrho^{\prime}-\kappa, \quad \varrho^{2}=\varrho-\kappa, \quad r^{2}=r+\kappa, \\
\kappa=\varrho \varrho^{\prime}, \quad 1=\varrho+\varrho^{\prime} .
\end{gathered}
$$

The Bernstein inequality implies, for $x, y \in \mathbb{C}$, that

$$
|k(y)| \leq \kappa(\max \{1,|y| / r\})^{2}, \quad|l(x)| \leq \kappa(\max \{1,|x| / r\})^{2} .
$$

From the Cauchy inequality we deduce

$$
\begin{aligned}
& |f|,|F| \leq \kappa, \\
& |e|,|E| \leq \kappa / r, \\
& |c|,|C| \leq \kappa / r^{2} .
\end{aligned}
$$

We distinguish between three regions in $\mathbb{C}^{2}$ :

$$
\begin{aligned}
G_{0} & :=\left\{(x, y) \in \mathbb{C}^{2}:|x|,|y|<\varrho\right\}, \\
G_{\infty} & :=\left\{(x, y) \in \mathbb{C}^{2}: \max \{|x|,|y|\}>r\right\}, \\
G_{1} & :=\left\{(x, y) \in \mathbb{C}^{2}: \varrho \leq \max \{|x|,|y|\} \leq r\right\} .
\end{aligned}
$$

$G_{0}$ is mapped to itself as, for $|x|,|y|<\varrho$, one calculates that $\left|x^{2}+k(y)\right|$ and $\left|y^{2}+l(x)\right|$ are bounded by $\varrho$. We deduce that the family $\left\{\left.f^{k}\right|_{G_{0}}\right\}$ of iterates of $f$ restricted to $G_{0}$ is normal, hence

$$
G_{0} \subseteq F(f) .
$$

Similar calculations show that $G_{\infty}$ is also mapped to itself and that the family $\left\{\left.f^{k}\right|_{G_{\infty}}\right\}$ converges to infinity. We obtain the inclusion

$$
J(f) \subseteq \partial K(f) \subseteq G_{1} .
$$

Now we divide the remaining set $G_{1}$ into the following sets:

$$
\begin{aligned}
G_{1 x y} & :=\left\{(x, y) \in \mathbb{C}^{2}: \varrho \leq|x|,|y| \leq r\right\}, \\
G_{1 x} & :=\left\{(x, y) \in \mathbb{C}^{2}: \varrho \leq|x| \leq r,|y|<\varrho\right\}, \\
G_{1 y} & :=\left\{(x, y) \in \mathbb{C}^{2}:|x|<\varrho, \varrho \leq|y| \leq r\right\} .
\end{aligned}
$$


From the above we deduce that $f$ maps points from $G_{1 x}$ to $G_{1 x}, G_{0}$, or $G_{\infty}$. Points of $G_{1 y}$ are mapped into $G_{1 y}, G_{0}$, or $G_{\infty}$. Clearly,

$$
f^{-1}\left(G_{1 x y}\right) \subseteq G_{1 x y}
$$

and

$$
f^{-1}\left(G_{1 x}\right) \subseteq G_{1 x} \dot{\cup} G_{1 x y}, \quad f^{-1}\left(G_{1 y}\right) \subseteq G_{1 y} \dot{\cup} G_{1 x y} .
$$

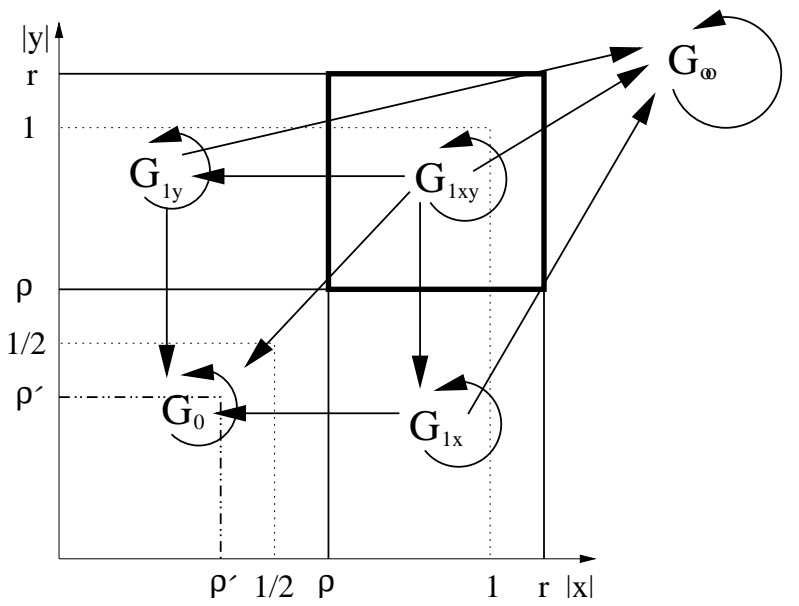

Fig. 1. Sketch (Reinhardt diagram) of the regions $G_{*}$

The critical points of $f$ (where $f$ does not have full rank) are given by the zeros of the Jacobi determinant. Namely, a critical point $(x, y)$ satisfies the equations

$$
\begin{aligned}
(2(1-c C) y-C e) x & =c E y, \\
(2(1-c C) x-c E) y & =C e x .
\end{aligned}
$$

This immediately yields the following lemma.

LEMMA 3.5. The critical points and their forward images are bounded away from $G_{1 x y}$.

P r o o f. Elementary calculations.

Corollary 3.6. In $G_{1 x y}$ all inverse branches $\left.f_{*}^{-k}\right|_{G_{1 x y}}: G_{1 x y} \rightarrow G_{1 x y}$ of $f^{k}$ are well-defined.

An analogue to (13) still holds if we replace $G_{1 x y}$ by an open neighbourhood $\widetilde{G}_{1 x y}$ which is the product of two ring domains whose inner (resp. outer) radius is slightly smaller than $\varrho$ (resp. slightly greater than $r$ ). Introducing the hyperbolic metric on $\widetilde{G}_{1 x y}$ (cf. [18]) we see that (in this metric) $D f^{-1}$ must be strictly contracting on the compact set $G_{1 x y}$. We deduce that 
there exists $\Theta>0$ such that on $G_{1 x y}$, for some $\lambda<1$,

$$
\left\|D f^{-k}\right\| \leq \Theta \lambda^{k}
$$

since, on $G_{1 x y}$, the Euclidean metric is equivalent to the hyperbolic metric. This means that $D f^{-k}$ is contracting with factor $\Theta \lambda^{k}$, and $D f^{k}$ is expanding by at least $1 /\left(\Theta \lambda^{k}\right)$ on $G_{1 x y}$.

From $f^{-1}\left(\bar{B}_{R_{f}}\right) \subseteq \bar{B}_{R_{f}}$ we derive that

$$
K(f)=\lim _{k \rightarrow \infty} f^{-k}\left(\bar{B}_{R_{f}}\right) .
$$

Moreover, we directly see that

$$
(\partial K)^{*}:=\lim _{k \rightarrow \infty} f^{-k}\left(G_{1 x y}\right)
$$

is a completely invariant set and a repeller under iteration of $f$. Furthermore, it is easy to see that, for any point $z^{*} \in G_{1 x y}$, we have

$$
\overline{\lim _{k \rightarrow \infty} f^{-k}\left(z^{*}\right)}=(\partial K)^{*} \text {. }
$$

This implies that

$$
(\partial K)^{*}=\lim _{k \rightarrow \infty} f^{-k}\left(\partial_{\mathrm{SH}} \bar{B}_{R_{f}}\right) .
$$

We want to investigate the topological structure of $(\partial K)^{*}$ and the dynamics of $f$ on $(\partial K)^{*}$. We define a family of maps parametrised by $t \in[0,1]$ by setting

$$
f_{t}(z):=(1-t) \sigma_{2}(z)+t f(z)
$$

From the above considerations it follows that for each of these maps we can find a repeller $(\partial K)_{t}^{*}$ which is contained in $G_{1 x y}$. Expanding repellers are structurally stable and the conjugacies near the identity depend continuously on the parameter. It follows that there exist homeomorphisms $\pi_{t}$, continuously depending on $t$, such that the following diagram is commutative:

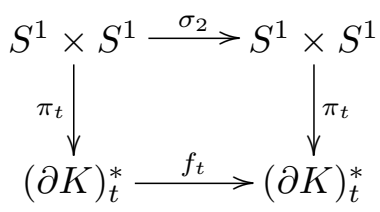

We have shown the following theorem:

THEOREM 3.7. There exists an isotopy from the identity map on $S^{1} \times S^{1}$ to $\pi_{1}: S^{1} \times S^{1} \rightarrow(\partial K)^{*}$ in $G_{1 x y}$. Moreover, $\left((\partial K)^{*}, f\right)$ is a mixing repeller.

Proof. The isotopy can be established via the maps $\pi_{t}$. The mixing property then follows from the mixing property of $\left(S^{1} \times S^{1}, \sigma_{2}\right)$.

We now compare $(\partial K)^{*}$ with the Shilov boundary of $K$. 
Lemma 3.8. Let $K_{i}, i \in \mathbb{N}$, be a sequence of nonempty compact polynomially convex sets in $\mathbb{C}^{n}$ such that $K_{i+1} \subseteq K_{i}$. Let

$$
K_{\infty}:=\lim _{i \rightarrow \infty} K_{i}=\bigcap_{i=1}^{\infty} K_{i} .
$$

Then

$$
\partial_{\mathrm{SH}} K_{\infty} \subseteq \lim _{i \rightarrow \infty} \partial_{\mathrm{SH}} K_{i} .
$$

Pr o of. Let $z^{*} \in \partial_{\mathrm{SH}} K_{\infty}$. Hence for $\eta>0$ and $1 / 2>\delta>0$ there exists a function $\vartheta_{\eta, z^{*}} \in \mathbb{A}$ such that

$$
\begin{aligned}
\sup _{z \in K_{\infty}}\left|\vartheta_{\eta, z^{*}}(z)\right|= & \sup _{z \in B_{\eta / 2}\left(z^{*}\right) \cap K_{\infty}}\left|\vartheta_{\eta, z^{*}}(z)\right|=1, \\
& \sup _{z \in \mathrm{C} B_{\eta / 2}\left(z^{*}\right) \cap K_{\infty}}\left|\vartheta_{\eta, z^{*}}(z)\right|<\delta / 4 .
\end{aligned}
$$

We choose a holomorphic map $\vartheta_{\eta, z^{*}}^{0} \in \mathbb{A}_{0}$ defined on some closed neighbourhood $\overline{B_{\iota}\left(K_{\infty}\right)}$ for which

$$
\sup _{z \in K_{\infty}}\left|\vartheta_{\eta, z^{*}}(z)-\vartheta_{\eta, z^{*}}^{0}(z)\right|<\delta / 4
$$

$\vartheta_{\eta, z^{*}}^{0}$ is still a peak function for $B_{\eta / 2}\left(z^{*}\right)$, since

$$
\begin{gathered}
\sup _{z \in B_{\eta / 2}\left(z^{*}\right) \cap K_{\infty}}\left|\vartheta_{\eta, z^{*}}^{0}(z)\right| \geq 1-\delta / 4, \\
\sup _{z \in \mathrm{C} B_{\eta / 2}\left(z^{*}\right) \cap K_{\infty}}\left|\vartheta_{\eta, z^{*}}^{0}(z)\right|<\delta / 4+\delta / 4 .
\end{gathered}
$$

By equicontinuity of $\vartheta_{\eta, z^{*}}^{0}$ on $\overline{B_{\iota}\left(K_{\infty}\right)}$ we deduce that there exists $\tau>0$ such that, for $z \in B_{\tau}\left(K_{\infty}\right)$, we have

$$
\begin{aligned}
\sup _{z \in B_{\tau}\left(K_{\infty}\right) \backslash B_{\eta}\left(z^{*}\right)}\left|\vartheta_{\eta, z^{*}}^{0}(z)\right|<3 \delta / 4, \\
\sup _{z \in B_{\eta}\left(z^{*}\right) \cap K_{\infty}}\left|\vartheta_{\eta, z^{*}}^{0}(z)\right|>1-\delta / 2 .
\end{aligned}
$$

Since $K_{\infty}=\lim _{i \rightarrow \infty} K_{i}$ we can find an index $i^{*}$ such that, for $i \geq i^{*}, K_{i}$ is contained in $B_{\tau}\left(K_{\infty}\right)$. Then the function $\vartheta_{\eta, z^{*}}^{0}$ restricted to $K_{i}$ (where $\left.i \geq i^{*}\right)$ must take its maximal modulus in $\partial_{\mathrm{SH}} K_{i}$. From (18) and (19) we deduce that $\partial_{\mathrm{SH}} K_{i} \cap B_{\eta}\left(z^{*}\right) \neq \emptyset$, hence

$$
\partial_{\mathrm{SH}} K_{\infty} \subseteq \lim _{i \rightarrow \infty} \partial_{\mathrm{SH}} K_{i} .
$$

COROllary 3.9. The Shilov boundary of $K(f)$, which is contained in $J(f)$ by Theorem 3.1, is contained in the limit of the inverse images of the Shilov boundary of $\bar{B}_{R_{f}}$ :

$$
\partial_{\mathrm{SH}} K(f) \subseteq(\partial K)^{*}=\lim _{k \rightarrow \infty} f^{-k}\left(\partial_{\mathrm{SH}} \bar{B}_{R_{f}}\right) .
$$


Proof (cf. Theorem 2.4). The sets $f^{-k}\left(\bar{B}_{R_{f}}\right)$ are Weil analytic polyhedra defined by

$$
\varphi_{k, 1}(x, y):=\frac{1}{R_{f}} \pi_{1} \circ f^{k}(x, y), \quad \varphi_{k, 2}(x, y):=\frac{1}{R_{f}} \pi_{2} \circ f^{k}(x, y),
$$

hence

$$
\partial_{\mathrm{SH}} f^{-k}\left(\bar{B}_{R_{f}}\right)=f^{-k}\left(\partial_{\mathrm{SH}} \bar{B}_{R_{f}}\right) .
$$

Now set $K_{i}:=f^{-i}\left(\bar{B}_{R_{f}}\right)$ in Lemma 3.8.

We shall prove that in (20) we actually get equality of $\partial_{\mathrm{SH}} K(f)$ and $(\partial K)^{*}$.

Lemma 3.10. For two disjoint compact disks $D, E \subseteq \mathbb{B}$ there exists a holomorphic function $\varphi: \mathbb{B} \rightarrow \mathbb{B}$ such that for given $1 / 2>\varrho>0$,

$$
\begin{aligned}
& \inf _{z \in E}|\varphi(z)| \geq 1-\varrho, \\
& \sup _{z \in D}|\varphi(z)| \leq \varrho .
\end{aligned}
$$

Pr o of. Without loss of generality we can assume that

$$
D=\overline{B_{\sigma}(s)}, \quad E=\overline{B_{\tau}(t)}
$$

with $s, t \in[0,1], s<t$, and $\sigma, \tau>0$, such that $D, E \subset \mathbb{B}$ and $D \cap E=\emptyset$. For some $\eta, \vartheta>0$,

$$
E \subseteq B_{\eta}(1), \quad D \cap B_{\eta+\vartheta}(1)=\emptyset .
$$

We realize $\varphi$ as a mapping of the following form: For $\delta, \kappa>0$ we define

$$
\varphi_{\delta, \kappa}: z \mapsto\left(\frac{1+\delta-z}{\delta}\right)^{-\kappa} .
$$

In order to obtain (21) and (22) it is sufficient to choose $\delta$ and $\kappa$ such that

$$
\left(1+\frac{\eta}{\delta}\right)^{-\kappa} \geq-\varrho
$$

and

$$
\left(1+\frac{\eta+\vartheta}{\delta}\right)^{-\kappa} \leq \varrho
$$

We deduce that

$$
\frac{\eta+\vartheta}{\sqrt[-\kappa]{\varrho}-1} \leq \delta \leq \frac{\eta}{\sqrt[-\kappa]{1-\varrho}-1},
$$

hence we have to choose $\kappa$ (depending on $\eta, \vartheta, \varrho$ ) such that

$$
\frac{\eta+\vartheta}{\vartheta} \leq \frac{\sqrt[-\kappa]{\varrho}-1}{\sqrt[-\kappa]{1-\varrho}-1}
$$


As for $0<\varrho<1 / 2$,

$$
\lim _{\kappa \searrow 0} \frac{\sqrt[-\kappa]{\varrho}-1}{\sqrt[-\kappa]{1-\varrho}-1}=\infty
$$

we can find such a $\kappa$ and can also choose a $\delta$ which satisfies (24).

COROLlary 3.11. For a finite set of pairwise disjoint compact nonempty bi-disks $B_{i}=D_{i} \times E_{i}, i=0, \ldots, r$, in $\mathbb{B} \times \mathbb{B}$ and any constant $0<\sigma<1 / 2$ there exists a holomorphic function $\varphi: \mathbb{B} \times \mathbb{B} \rightarrow \mathbb{B}$ such that

$$
\inf _{z \in B_{0}}|\varphi(z)| \geq 1-\sigma, \quad \sup _{\substack{z \in B_{i} \\ i=1, \ldots, r}}|\varphi(z)| \leq \sigma .
$$

Proof. Define $\varphi(z)$ as the product of functions $\psi_{i}(x, y)$, where $\psi_{i}(x, y)$ $:=\varphi_{i}(x)$ if $D_{0} \cap D_{i}=\emptyset$, otherwise $\psi_{i}(x, y):=\varphi_{i}(y)$.

REMARK 3.12. The generalisation to the case of higher-dimensional polydisks in $\mathbb{B}^{n}, n>2$, is evident. Furthermore, it is obvious that one can replace $\mathbb{B} \times \mathbb{B}$ by $B_{R_{f}}$.

TheOREM 3.13. For $z^{*} \in \partial_{\mathrm{SH}} K$, the whole backward orbit $\left\{f_{i}^{-k}\left(z^{*}\right)\right\}$ of $z^{*}$ is contained in $\partial_{\mathrm{SH}} K$ (where we index the $4^{k}$ different $k$ th inverse branches by integers $i$ ).

Proof. Fix one inverse branch $f_{0}^{-k}$ of $f^{k}$. For $U \ni f_{0}^{-k}\left(z^{*}\right)$ we find an open set $V \ni z^{*}$ such that $f_{0}^{-k}(V) \subseteq U$ and, for all inverse branches, the images $f_{i}^{-k}(V)$ are contained in disjoint bi-disks $D_{i} \times E_{i} \subseteq B_{R_{f}}$. For $V$ we have a peak function $\varphi_{V}$. For the $D_{i} \times E_{i} \subseteq B_{R_{f}}$ we can construct $\varphi$ as in Corollary 3.11. Then

$$
\Phi_{U}: z \mapsto \varphi(z) \cdot \varphi_{V} \circ f^{k}(z)
$$

is a peak function for $U$.

As $\partial_{\mathrm{SH}} K(f) \neq \emptyset$ there exists at least one point $z^{*} \in \partial_{\mathrm{SH}} K(f)=$ $\partial_{\mathrm{SH}} K(f) \cap(\partial K)^{*}$. Since its backward images are dense in $(\partial K)^{*}$, we have shown

TheOREM 3.14. The Shilov boundary of $K(f)$ equals the set of limit points of $\left(f^{-k}\left(\partial_{\mathrm{SH}} \bar{B}_{R_{f}}\right)\right)_{k \in \mathbb{N}}$ :

$$
\partial_{\mathrm{SH}} K(f)=(\partial K)^{*} .
$$

In order to determine $J(f)$ completely it remains to show that $\partial K(f) \backslash$ $(\partial K)^{*} \subset F(f)$. Note that, for $z \in G_{1 x y}$, either $z \in(\partial K)^{*}$ or $f^{k}(z)$ eventually leaves $G_{1 x y}$ (and stays outside according to (13)). We know that $G_{0} \cup G_{\infty} \subseteq$ $F(f)$. Thus we are left with $z \in G_{1 x} \cup G_{1 y}$ or $z \in G_{1 x y}$, which are eventually mapped to $G_{1 x} \cup G_{1 y}$ and whose forward orbits stay in this set. 
In our treatment of this case $z^{*} \in G_{1 y}, G_{1 x}$, resp., we apply [12], pp. 271 $\mathrm{ff}$., in the special case of a torus map. In order to do so we need some additional notation.

For some $\eta>0$ let

$$
e=(w, x, y) \in B_{\eta}(0,0,0) \subseteq \mathbb{C}^{3} .
$$

(We will use $B_{\eta}(0,0)$ for balls in $\mathbb{C}^{2}, B_{\eta}(0)$ in $\mathbb{C}^{1}$, etc.) Assume that we are given a sequence of holomorphic maps

$$
T_{n}: B_{\eta}(0,0,0) \rightarrow \mathbb{C}^{3},
$$

with

$$
T_{0}(e)=e, \quad T_{n}(e):=\left(\begin{array}{r}
U_{n}(w) \\
A_{n} x+F_{n}(e) \\
B_{n} y+G_{n}(e)
\end{array}\right),
$$

where the complex constants $A_{n}, B_{n}$ satisfy the relations

$$
\left|A_{n}\right| \leq \alpha, \quad\left|B_{n}^{-1}\right| \leq 1 / \beta,
$$

with $\alpha<1$ and $\beta>1$, hence in particular $B_{n} \neq 0$ for all $n \geq 1$. Furthermore, let

$$
U_{n}(0)=0, \quad F_{n}(0,0,0)=0, \quad G_{n}(0,0,0)=0,
$$

and assume that, for all $w_{a}, w_{b} \in B_{\eta}(0)$,

$$
\left|U_{n}\left(w_{a}\right)-U_{n}\left(w_{b}\right)\right| \leq\left|w_{a}-w_{b}\right| ;
$$

finally, for some $0<\delta<1 / 10$ with

$$
0<\alpha<1-2 \delta<1+5 \delta<\beta,
$$

and for all $e_{a}, e_{b} \in B_{\eta}(0,0,0)$, the relations

$$
\left.\begin{array}{r}
\left|F_{n}\left(e_{a}\right)-F_{n}\left(e_{b}\right)\right| \\
\left|G_{n}\left(e_{a}\right)-G_{n}\left(e_{b}\right)\right|
\end{array}\right\} \leq \delta^{2}\left\|e_{a}-e_{b}\right\|
$$

shall hold. We obtain a second sequence of maps by setting

$$
S_{n}:=T_{n} \circ \ldots \circ T_{1} .
$$

We define its stability set $\mathcal{D}$ with respect to $B_{\eta}(0,0,0)$ as

$$
\mathcal{D}:=\bigcap_{n=1}^{\infty} \mathcal{D}_{n},
$$

where $\mathcal{D}_{n}$ denotes the set where $S_{n}$ is actually defined.

Then the following theorem holds.

THEOREM $3.15([12])$. Under the above assumptions there exists a holomorphic map

$$
y_{0}: B_{\eta}(0,0) \rightarrow B_{\eta}(0)
$$


such that

$$
\mathcal{D}=\left\{(w, x, y): y=y_{0}(w, x) \text { on } B_{\eta}(0,0)\right\}
$$

If we set

$$
e_{0}:=\left(w_{0}, x_{0}, y_{0}\left(w_{0}, x_{0}\right)\right)
$$

for $\left(w_{0}, x_{0}\right) \in B_{\eta}(0,0)$, and

$$
e_{k}:=S_{k}\left(e_{0}\right)=\left(w_{k}\left(x_{0}, w_{0}\right), x_{k}\left(x_{0}, w_{0}\right), y_{k}\left(w_{0}, x_{0}\right)\right),
$$

then for $k \geq 0$,

$$
e_{k}=(0,0,0)
$$

holds if and only if $w_{0}=x_{0}=0$. Furthermore, for another pair $\left(w^{0}, x^{0}\right) \in$ $B_{\eta}(0,0)$ we get

$$
\begin{aligned}
\mid y_{k}\left(w^{0}, x^{0}\right)- & y_{k}\left(w_{0}, x_{0}\right) \mid \\
\leq & (1-2 \delta)\left(\delta\left|w^{0}-w_{0}\right|+\left|x_{k}\left(w^{0}, x^{0}\right)-x_{k}\left(w_{0}, x_{0}\right)\right|\right),
\end{aligned}
$$

and

$$
\left|x_{k}\left(w^{0}, x^{0}\right)-x_{k}\left(w_{0}, x_{0}\right)\right| \leq \delta\left|w^{0}-w_{0}\right|+\left(\alpha+2 \delta^{2}\right)^{k}\left|x^{0}-x_{0}\right| .
$$

Moreover, $y=y_{0}(w, x)$ is "invariant" on $B_{\eta}(0,0)$, i.e.

$$
y_{k}\left(w_{0}, x_{0}\right) \equiv y_{0}\left(w_{k}\left(w_{0}, x_{0}\right), x_{k}\left(w_{0}, x_{0}\right)\right) .
$$

Evidently, $\mathcal{D}$ is a possible choice for $\mathcal{C}_{0}$ if we want to show weak normality of the family $\left\{S_{n}\right\}$ at $(0,0,0)$.

We shall show that the iterates of a torus map around a point in $G_{1 x}$ or $G_{1 y}$ can be embedded in a family of mappings $S_{n}$ in $\mathbb{C}^{3}$ such that the above conditions are satisfied.

We define

$$
\varrho^{*}:=\frac{1}{2}-\frac{1}{2} \sqrt{\varepsilon}
$$

then

$$
G_{1 y}^{*}:=\left\{(x, y): \varrho \leq|y| \leq r,|x|<\varrho^{*}\right\} \subset G_{1 y},
$$

and analogously $G_{1 x}^{*} \subset G_{1 x}$. Since $\varrho^{\prime}<\varrho^{*}<\varrho, z \in G_{1 y}$ is eventually mapped into $G_{1 y}^{*}$ and stays there or enters $G_{0} \cup G_{\infty}$, and analogously for $y$ replaced by $x$.

Assume that for a $z^{*}=\left(x_{0}^{*}, y_{0}^{*}\right)$ and all $n \in \mathbb{N}$,

$$
\left(x_{n}^{*}, y_{n}^{*}\right):=f^{n}\left(x_{0}^{*}, y_{0}^{*}\right) \in G_{1 y}^{*} .
$$

Define a sequence of polynomial maps $T_{n}$ on $B_{\eta}(0,0,0)$ according to 3.15:

$$
\begin{aligned}
T_{0}(w, x, y) & :=\left(0, x_{0}^{*}, y_{0}^{*}\right)+(w, x, y), \\
T_{n+1}(w, x, y) & :=\left(w, f\left(\left(x_{n}^{*}, y_{n}^{*}\right)+(x, y)\right)-\left(x_{n+1}^{*}, y_{n+1}^{*}\right)\right) .
\end{aligned}
$$


For $n \geq 1$, these maps have the form

$$
T_{n}(x, y)=\left(\begin{array}{l}
w \\
A_{n} x+F_{n}(x, y) \\
B_{n} y+G_{n}(x, y)
\end{array}\right)
$$

where $A_{n}=2 x_{n}^{*}, B_{n}=2 y_{n}^{*}$, and

$$
\begin{aligned}
& F_{n}(x, y)=\left(e+2 c y_{n}^{*}\right) y+x^{2}+c y^{2}, \\
& G_{n}(x, y)=\left(E+2 C x_{n}^{*}\right) x+y^{2}+C x^{2} .
\end{aligned}
$$

We can choose $\alpha:=2 \varrho^{*}$ and $\beta:=2 \varrho$. For $e_{a}=\left(w_{a}, x_{a}, y_{a}\right), e_{b}=\left(w_{b}, x_{b}, y_{b}\right)$ $\in B_{\eta}(0,0,0)$ we obtain the estimates

$$
\left|F_{n}\left(e_{a}\right)-F_{n}\left(e_{b}\right)\right| \leq\left(\kappa / r+2 \kappa / r+2 \eta+2 \kappa \eta / r^{2}\right)\left\|e_{a}-e_{b}\right\|,
$$

and

$$
\left|G_{n}\left(e_{a}\right)-G_{n}\left(e_{b}\right)\right| \leq\left(\kappa / r+2 \kappa \varrho^{*} / r^{2}+2 \eta+2 \kappa \eta / r^{2}\right)\left\|e_{a}-e_{b}\right\| .
$$

If we choose $\varepsilon$ large enough, say $\varepsilon>\varepsilon_{2}$, and $\eta$ small, we see that, for all $e_{a}, e_{b}$ in $B_{\eta}(0,0,0)$ and $n \in \mathbb{N}^{*}$,

$$
\left.\begin{array}{r}
\left|F_{n}\left(e_{a}\right)-F_{n}\left(e_{b}\right)\right| \\
\left|G_{n}\left(e_{a}\right)-G_{n}\left(e_{b}\right)\right|
\end{array}\right\}<\delta^{2}\left\|e_{a}-e_{b}\right\|
$$

where we can choose $\delta$ such that $0<\delta<\min \{1 / 10,2 \sqrt{\varepsilon} / 5\}$. Then, for all $n \in \mathbb{N}^{*}$,

$$
\begin{aligned}
& \left|A_{n}\right|<1-2 \delta, \\
& \left|B_{n}\right|>1+5 \delta .
\end{aligned}
$$

Hence, we can apply Theorem 3.15. We have constructed $T_{n}$ such that

$$
S_{n}(w, x, y)=\left(w, f^{n}\left(z^{*}+z\right)-f^{n}\left(z^{*}\right)\right),
$$

hence the stability set $\mathcal{D}$ (dependent on $z^{*}$ ) yields $\mathcal{C}_{z^{*}}$, hence $z^{*} \in F(f)$. We have shown that $(\partial K)^{*}=J(f)$. The following proposition holds.

Proposition 3.16. For a torus map, (A), (B), and (D) are equivalent definitions for the Julia set.

We are left with (C) and (E). We shall start with (E).

The argument given in [15] for the construction of the Green function $G_{K}$ applies. We see that

$$
G_{K}(f(z))=2 G_{K}(z) .
$$

If we define the measure $\mu_{K}$ as $\left(d d^{C} G_{K}\right)^{2}$, then we get

$$
\mu_{K} \circ f=2^{2} \mu_{K} \text {. }
$$

It is obvious that $\mu_{K}$ is $f$-invariant and has entropy $2 \log (2)$. By Gromov's result [11] this is equal to the topological entropy of $f$. We conclude that 
$\mu_{K}$ has maximal entropy. Since we already know that the Shilov boundary and the Julia set are equal, we have shown that $J(f)$ is the support of a measure of maximal entropy for $f$.

REMARK 3.17. We note that the map $\pi_{1}$ from (17) can be used to transport the (normalised) Lebesgue measure on $S^{1} \times S^{1}$ to $J(f)$ and thereby obtain an invariant measure (by virtue of (17)) which has maximal entropy. It equals $\mu_{K}$ by uniqueness of the measure of maximal entropy for mixing repellers.

Use of (17) and the fact that repelling periodic points of $\sigma_{2}$ are dense in $S^{1} \times S^{1}$ shows that the repelling periodic points of $f$ are dense in $J(f)$. We derive that

$$
J(f) \subseteq \overline{\{z: z \text { is a repelling periodic point of } f\}} .
$$

Clearly any periodic point in $G_{0}$ must be attracting and $G_{\infty}$ contains $\infty$ as the only attracting periodic point. The equivalence of $(\mathrm{C})$ to the other definitions of $J(f)$ follows if we can show that there are no repelling periodic points in $G_{1 x}$ and $G_{1 y}$. But in the case of a fixed point $z^{*}$ of $f^{k}$ in one of

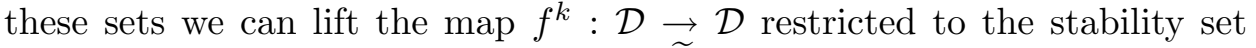
$\mathcal{D}$ of $z^{*}$ to a self-map of the unit disk: $\widetilde{f}: \mathbb{B} \rightarrow \mathbb{B}$ with fixed point 0 . The derivative of $\widetilde{f}$ at 0 has modulus at most 1 according to the lemma of Pick ([19], p. 194). This contradicts $\left\|\left(D\left(f^{k}\right)\right)^{-1}\right\|<1$ for $z^{*}$. We have shown the equivalence of $(\mathrm{C})$ to the other definitions of $J(f)$ for a torus map.

\section{References}

[1] J. Aaronson, M. Denker, and M. Urbański, Ergodic theory of Markov fibred systems and parabolic rational maps, Trans. Amer. Math. Soc. 337 (1993), 495-548.

[2] A. F. Beardon, Iteration of Rational Functions, Grad. Texts in Math. 132, Springer, 1991.

[3] E. Bedford and J. Smillie, Polynomial diffeomorphisms of $\mathbb{C}^{2}$ : currents, equilibrium measure and hyperbolicity, Invent. Math. 103 (1991), 69-99.

[4] H. Brolin, Invariant sets under iteration of rational functions, Ark. Mat. 6 (1965), 103-144.

[5] J. E. Fornæss and N. Sibony, Complex dynamics in higher dimension. I, Astérisque 222 (1994), 201-231.

[6] O. Forster, Riemannsche Flächen, Heidelberger Taschenbücher 184, Springer, 1977.

[7] B. A. Fuks, Special Chapters in the Theory of Analytic Functions of Several Variables, Transl. Math. Monographs 14, Amer. Math. Soc., 1991.

[8] I. R. Gelfand, D. A. Raikow und G. E. Schilow, Kommutative normierte Algebren, Deutscher Verlag der Wiss., 1964.

[9] H. Grauert und K. Fritzsche, Einführung in die Funktionentheorie mehrerer Veränderlicher, Hochschultext, Springer, 1974.

[10] H. Grauert and R. Remmert, Coherent Analytic Sheaves, Springer, 1984. 
[11] M. Gromov, On the entropy of holomorphic mappings, preprint, Inst. Hautes Etudes Sci.

[12] P. Hartman, Ordinary Differential Equations, Birkhäuser, 1982.

[13] S. M. Heinemann, Iteration holomorpher Abbildungen in $\mathbb{C}^{n}$, Diplomarbeit Universität Göttingen, 1993.

[14] —, Dynamische Aspekte holomorpher Abbildungen in $\mathbb{C}^{n}$, Dissertation, Universität Göttingen, 1994.

[15] - Julia sets for endomorphisms of $\mathbb{C}^{n}$, Ergodic Theory Dynam. Systems 16 (1996), $1275-1296$.

[16] K. Knopp, Elemente der Funktionentheorie, Sammlung Göschen 2124, de Gruyter, 1978.

[17] K. Krzyżewski and W. Szlenk, On invariant measures for expanding differentiable mappings, Studia Math. 33 (1969), 83-92.

[18] S. Lang, Introduction to Complex Hyperbolic Spaces, Springer, 1987.

[19] R. Remmert, Funktionentheorie I, Grundwissen Math. 5, Springer, 1984.

[20] G. Scheja und U. Storch, Lehrbuch der Algebra 2, Mathematische Leitfäden, Teubner, 1988.

[21] F. Schweiger, Numbertheoretical endomorphisms with $\sigma$-finite invariant measure, Israel J. Math. 21 (1975), 308-318.

[22] I. R. Shafarevich, Basic Algebraic Geometry, Springer, 1974.

Institut für Mathematische Stochastik

Georg-August-Universität Göttingen

Lotzestraße 13

D-37083 Göttingen, Germany

E-mail: denker@math.uni-goettingen.de

sheinema@math.uni-goettingen.de

Received 22 October 1997;

in revised form 14 April 1998 